\title{
Attractor behaviour in ELKO cosmology
}

\author{
Abhishek Basak, ${ }^{a, 1}$ Jitesh R. Bhatt ${ }^{a}$ S. Shankaranarayanan ${ }^{b}$ and K. \\ V. Prasantha Varma ${ }^{b}$ \\ ${ }^{a}$ Theoretical Physics Group, Physical Research Laboratory, Ahmedabad, India \\ ${ }^{b}$ Indian Institute of Science Education and Research-Thiruvananthapuram (IISER-TVM), \\ Trivandrum 695016, India \\ E-mail: abhishek@prl.res.in, jeet@prl.res.in, shanki@iisertvm.ac.in, \\ varma@iisertvm.ac.in
}

Abstract. We study the dynamics of ELKO in the context of accelerated phase of our universe. To avoid the fine tuning problem associated with the initial conditions, it is required that the dynamical equations lead to an early-time attractor. In the earlier works, it was shown that the dynamical equations containing ELKO fields do not lead to early-time stable fixed points. In this work, using redefinition of variables, we show that ELKO cosmology admits early-time stable fixed points. More interestingly, we show that ELKO cosmology admit two sets of attractor points corresponding to slow and fast-roll inflation. The fast-roll inflation attractor point is unqiue for ELKO as it is independent of the form of the potential. We also discuss the plausible choice of interaction terms in these two sets of attractor points and constraints on the coupling constant.

\footnotetext{
${ }^{1}$ Corresponding author.
} 


\section{Contents}

1 Introduction $\quad 1$

2 Background equations $\quad 2$

3 Slow-roll parameters for ELKOs $\quad 3$

4 Dynamical equations $\quad 4$

5 Fixed points and stability analysis: General Analysis 5

5.1 Case I 6

$\begin{array}{lll}5.2 & \text { Case II } & 7\end{array}$

6 Special cases of the interaction term $\quad 8$

6.1 Case I: Slow-roll $\quad 8$

6.1.1 $Q_{1}=\beta v^{2} x \quad 8$

6.1.2 $Q_{1}=\beta v^{2} x^{2} \quad 8$

6.1.3 $Q_{1}=\beta v x^{2} \quad 9$

6.2 Case II: Fast roll 9

6.2.1 $Q_{1}=\beta v^{2} x \quad 9$

6.2.2 $Q_{1}=\beta v^{2} x^{2} \quad 9$

$\begin{array}{ll}6.2 .3 Q_{1}=\beta v x^{2} & 10\end{array}$

$\begin{array}{lll}7 & \text { Conclusion } & 10\end{array}$

8 Acknowledments $\quad 10$

\section{Introduction}

Inflation is currently one of the successful paradigm of the early universe[1,2]. The success of inflation not only rests on solving the problems of the Friedman-Robertson-Walker model, and that it generates the primordial spectra of scalar (density) and tensor (gravitational waves) perturbations. The temperature variations of CMB as measured in WMAP and PLANCK - to a large extent - confirm that the these primordial density perturbations are generated due to rapid expansion of the quantum fluctuations in the early universe.

Current CMB measurements can at-most provide two physical quantities during inflation: (i) inflaton (dynamical field that dominates during inflation) potential and (ii) the first derivative of the potential in the observable scales [3]. It is still unclear what are the properties of the dynamical field that drive inflation. It is usually assumed that the field that dominates is a fundamental scalar field. While it is the simplest, recently, there has been a surge of activity to look at the plausibility that the inflaton has an internal structure [4-6]. One of the key results of the spinor condensate models compared to the standard inflationary models is the prediction of running of spectral index in the slow-roll limit that is consistent with the current CMB measurements [7].

Even if the observations provide us information about the nature of the dynamical field that drive inflation, the initial state of the field will never be known [8-10]. Einstein's 
equations are non-linear, hence, it is important to know what range of initial conditions of inflation can plausibly lead to inflation. For instance, it is possible that if the initial velocities of the background field are large then this will stop inflation [11].

The knowledge of initial conditions can provide crucial information about the nature of the fields and their interactions with the known matter fields [11, 12]. For instance, it is usually assumed that the inflaton is a fundamental scalar field. However, we do not know the nature of the scalar fields or its interaction with other fields. Similarly, it is not clear what are the properties of ELKOs and how they interact with the other fields. If the observations do provide evidence that the inflation occured due to the one of these fields, the initial conditions of these fields will provide information about the nature of interactions with standard model particles. This in turn can be useful for model building which can be verified in high-energy experiments.

The above issues are relevant and imperative to the current acceleration of the universe. It is unclear what dynamical fields drive the current accelerating universe. Even if the observations reveal the nature of the field, as in the case of inflation, it is not possible to know the initial condition that lead to current accelearation. This is referred to as cosmic coincidence problem. The constraints on the interaction of these fields with standard model particles will provide information about the initial condition that lead to acceleration.

In this work we investigate the following questions: If dynamical field during inflation is a condensate of the non-standard spinor whether a large set of initial conditions lead to inflation. If it does, then, can it constraint the interactions between the spinor fields and the standard model particles. Recently a spin-half fermion called ELKO was proposed as a candidate of dark matter $[13,14]$. Recently. in the literature several authors have shown that these can lead to accelerated expansion [7, 15-20].

In Ref. [21, 22], the authors could not find any stable fixed point with various kind of potentials. One of the draw-backs of their analyses is the choice of variables. Specifically, they have assumed $\phi$ and $V(\phi)$ to be independent variables. In this work, we make a combination of $\dot{\phi}, H$ and $V(\phi)$ and show that in these newly defined variables the dynamical equations have stable fixed points for a wide class of potentials and interactions between ELKO and matter. More interestingly, we show that ELKO cosmology admit two sets of attractor points corresponding to slow and fast-roll inflation. We also discuss the constraints on the coupling constants that will lead to early-time attractor behavior.

The manuscript is organised as follows: In sections 2 and 3 we define the background equations and slow-roll parameters. In section 4 we define new sets of variables and rewrite the background equations in terms of these variables. A general analysis of fixed point behavior is presented in section 5 and section 6 contains the fixed point analysis for specific potentials. Finally, in section 7 , we summarize our results and also discuss its implications.

\section{Background equations}

The new class of spinors named Elko were proposed as a candidate of dark matter in Ref.[13, 14]. These spinors are eigen spinors of charge conjugation operator. These are non standard spinors(NSS) because unlike the standard spinors Elko spinors have mass dimension one and $(C P T)^{2}=-\mathbb{I}$. As a consequence these spinors follow Klein-Gordon equation. Elko spinors are also called 'dark' spinor as its dominant interaction with standard model particles is via Higgs and gravity. Apart from being considered as inflaton these spinors have drawn lots of 
interests in works of different authors[23-27]. The $\operatorname{NSS}(\lambda)$ and its dual $(\vec{\lambda})$ can be defined as

$$
\lambda=\phi(t) \xi, \quad \vec{\lambda}=\phi(t) \vec{\xi},
$$

where $\xi$ and $\vec{\xi}$ are constant spinors with the property $\vec{\xi} \xi=\mathbb{I}$, and $\phi(t)$ is a scalar which is time depepndent. The action can be written as [boeh]

$$
S=\int d^{4} x \sqrt{-g}\left[\frac{1}{2} g^{\mu \nu} \vec{\lambda} \overleftarrow{\nabla}_{(\mu} \nabla_{\nu)} \lambda-V(\vec{\lambda} \lambda)\right]
$$

where $\nabla_{\mu}$ is the covariant derivative and the round bracket in the subscript denotes symmetrisation. Varying the action (2.2) the energy density and pressure can be written in terms as

$$
\begin{aligned}
\rho_{\phi} & =\frac{1}{2} \dot{\phi}^{2}+\frac{3}{8} H^{2} \phi^{2}+V(\phi), \\
p_{\phi} & =\frac{1}{2} \dot{\phi}^{2}-\frac{1}{4}\left(H \phi^{2}\right) \cdot-\frac{3}{8} H^{2} \phi^{2}-V(\phi),
\end{aligned}
$$

where $H$ is the Hubble parameter. The equation of motion can be written as:

$$
\ddot{\phi}+3 H \dot{\phi}-\frac{3}{4} H^{2} \phi+V_{, \phi}=0
$$

\section{Slow-roll parameters for ELKOs}

Due to the presence of the $H^{2} \phi^{2}$ term in the density and pressure, one has be careful in defining the slow-roll parameters for the ELKO condensate. In this section, we give the expressions for the slow-roll parameters. From the expression of time-time component of Einstein's equation one can write the Friedman's equation for NSS as:

$$
H^{2}=\frac{1}{3 M_{p l}^{2}}\left(\frac{\dot{\varphi}^{2}}{2 D}+\hat{V}\right)
$$

where $D=1-\tilde{F}=1-\frac{\varphi^{2}}{8 M_{p l}^{2}}$ and $\hat{V}=\frac{V}{D}$. Taking the time derivative on the both the sides of equation (3.1) one can write the slow-roll parameter $\epsilon$ as

$$
\epsilon=-\frac{\dot{H}}{H^{2}}=\frac{3}{2} \frac{\dot{\phi}^{2} / D}{\dot{\phi}^{2} / 2 D+\hat{V}}+\frac{\dot{D}}{H D}=\epsilon_{\mathrm{can}}+\alpha .
$$

where $\alpha=\frac{\dot{D}}{H D} \ll 1$ is an extra parameter which appears in the definition of the slow-roll parameter for ELKOs and $\epsilon_{\text {can }}$ is the slow-roll parameter defined for the canonical single scalar field, $\epsilon_{\text {can }}=\frac{3}{2} \frac{\dot{\phi}^{2}}{\dot{\phi}^{2} / 2+V} \simeq \frac{3}{2} \frac{\dot{\phi}^{2}}{V}\left(\right.$ when $\left.\dot{\phi}^{2} \ll V\right)$.

Substituting $\left(\dot{\phi}^{2} / 2 D+\tilde{V}\right)$ from $(3.2)$ in (3.1) one can write

$$
\dot{\phi}^{2}=2 M_{p l}^{2} H^{2} D(\epsilon-\alpha) .
$$

Now in this case we define $\delta=\frac{\ddot{\phi}}{H \dot{\phi}}$. Taking the time derivative on both the sides of (3.3) we get,

$$
\frac{\ddot{\phi}}{H \dot{\phi}}=\delta=-\epsilon+\frac{\alpha}{2}+\frac{(\epsilon-\alpha)}{2 H(\epsilon-\alpha)}
$$


The last term can be dropped as it is the time derivative of the slow-roll parameters. Therefore finally one can write the definition of $\delta$ as:

$$
\delta=-\epsilon+\frac{\alpha}{2}=-\epsilon_{\mathrm{can}}-\frac{\alpha}{2} .
$$

A closer inspection of above expression immediately suggests that $\delta$ is negative definite. For canonical scalar field, it is positive definite. This has an important effect for the spectra of scalar perturbations [7].

\section{Dynamical equations}

The expressions of energy density and pressure (2.4) can be written in terms of newly defined variables $X$ and $\tilde{V}$ as following

$$
\rho_{\phi}=X+\tilde{V}, \quad p_{\phi}=X-\tilde{V},
$$

where

$$
\begin{aligned}
X & =\frac{1}{2} \dot{\phi}^{2}-\frac{1}{8}\left(H \phi^{2}\right)^{\cdot} \\
\tilde{V} & =\frac{1}{8}\left(H \phi^{2}\right)^{\cdot}+\frac{3}{8} H^{2} \phi^{2}+V(\phi) .
\end{aligned}
$$

Physically, $X$ refers to the kinetic energy of the condensate field and $\tilde{V}$ refers to the potential energy of the condensate. Note that $H \dot{\phi} \phi$ acts like a friction term while $\dot{H} \phi^{2}$ acts as an antifriction term. Elko dynamics crucial depends on which of these two terms dominate during inflation.

Friedmann equation can be written as

$$
H^{2}=\frac{\kappa^{2}}{3} \rho_{t o t}=\frac{\kappa^{2}}{3}\left(\rho_{\phi}+\rho_{m}\right)
$$

where $\rho_{m}$ is the matter density and $\kappa^{2}=8 \pi G$. Using equation (4.1) we can write the Friedmann equation(4.4) as

$$
x^{2}+y^{2}+v^{2}=1,
$$

where $x, y$ and $v$ can be defined as $x=\frac{\kappa \sqrt{X}}{\sqrt{3} H}, y=\frac{\kappa \sqrt{\tilde{V}}}{\sqrt{3} H}$ and $v=\frac{\kappa \sqrt{\rho_{m}}}{\sqrt{3} H}$. Now, if we consider that the matter and dark energy are interacting only with themselves then the continuity equation

$$
\dot{\rho}_{\text {tot }}+3 H\left(\rho_{\text {tot }}+p_{\text {tot }}\right)=0
$$

can be written as two separate equations

$$
\begin{gathered}
\dot{\rho}_{\phi}+3 H\left(\rho_{\phi}+p_{\phi}\right)=-Q, \\
\dot{\rho}_{\mathrm{m}}+3 H\left(\rho_{\mathrm{m}}+p_{\mathrm{m}}\right)=Q,
\end{gathered}
$$

where $Q$ is the interaction term. In terms of the variables $x, y, v$ equations $(4.7,4.8)$ can be written respectively as

$$
x^{\prime}=(\epsilon-3) x-\frac{\lambda}{H} \frac{y^{2}}{x}-\frac{Q_{1}}{x},
$$




$$
v^{\prime}=\left(\epsilon-\frac{3}{2} \gamma\right) v+\frac{Q_{1}}{v}
$$

Here' is the derivative with respect to time divided by $H, \epsilon=-\frac{\dot{H}}{H^{2}}$ and $\lambda=\frac{\dot{\tilde{V}}}{\tilde{V}}, Q_{1}=\frac{\kappa^{2} Q}{6 H^{3}}$. To derive the above equations we have used the relation $p_{m}=(\gamma-1) \rho_{m}$, where $\gamma$ takes the value either 1 or $\frac{4}{3}$ depending on whether the universe is filled with cold matter or radiation respectively. Derivative of the variable $y$ with respect to time give us

$$
y^{\prime}=\left(\epsilon+\frac{\lambda}{2 H}\right) y
$$

$\dot{H}$ can be written as

$$
\dot{H}=-\frac{\kappa^{2}}{2}\left[\rho_{\phi}+p_{\phi}+\rho_{m}+p_{m}\right]
$$

Therefore we have three dynamical equations (4.9), (4.10) and (4.11) with one constraint (4.5). It is important to contrast the above set of variables with those used earlier [21]. The two variables $X$ and $\tilde{V}$ are independent of each other. However, in Wei's analysis [21], the two variables $y$ and $u$ are not independent.

In the rest of this work, we study the stability of fixed points with these equations. We show that ELKOs show a new set of fixed points that can not be identified directly with the canonical scalar field.

\section{$5 \quad$ Fixed points and stability analysis: General Analysis}

Fixed points are those points where the dynamical variables stop evolving, i.e., at fixed point $(\bar{x}, \bar{y}, \bar{v})$ the time derivative of $x, y$ and $v$ are zero. At fixed points, dynamical equations (4.9, $4.10,4.11)$ can be written as:

$$
\begin{gathered}
(\bar{\epsilon}-3) \bar{x}-\frac{\lambda}{H} \frac{\bar{y}^{2}}{\bar{x}}-\frac{Q_{1}}{\bar{x}}=0 \\
\left(\bar{\epsilon}-\frac{3}{2} \gamma\right) \bar{v}+\frac{Q_{1}}{\bar{v}}=0 \\
\left(\bar{\epsilon}+\frac{\lambda}{2 H}\right) \bar{y}=0
\end{gathered}
$$

Eq. (5.3) leads to two set of fixed points:

1. Case I: $\bar{y}=0$ and $\bar{\epsilon} \neq-\frac{\lambda}{2 H}$

2. Case II: $\bar{y} \neq 0$ and $\bar{\epsilon}=-\frac{\lambda}{2 H}$

In the rest of this section, we will consider the above two cases for general interaction term $Q_{1}$. In the following section, we consider special cases for the interaction term and discuss the nature of fixed points. 


\subsection{Case I}

Substituting this value of $\bar{y}$ in equation (5.1) we get

$$
\bar{\epsilon}=3 \bar{x}^{2}+\frac{Q_{1}}{\bar{x}^{2}}
$$

The above form of $\epsilon$ gives crucial information about the class of interaction terms between the condensate and matter fields that can lead to attractor behavior. In particular, it immediately shows that $Q_{1} \propto x^{2}$ may not lead to stable attractor points. Also it provides an upper bound on the coupling constant. We discuss these in the next section.

General expression for $\epsilon$ can be written as

$$
\epsilon=-\frac{\dot{H}}{H^{2}}=\frac{3}{2} \gamma+\left(3-\frac{3}{2} \gamma\right) x^{2}-\frac{3}{2} \gamma y^{2} .
$$

Therefore at fixed points $\bar{\epsilon}$ can be written as $\bar{\epsilon}=\frac{3}{2} \gamma+\left(3-\frac{3}{2} \gamma\right) \bar{x}^{2} . \bar{\epsilon}$ is a positive quantity ensuring accelerated expansion of the universe. Finally one can write an important relation for $\bar{\epsilon}$ which will be used later

$$
\bar{\epsilon}-3=\left(\frac{3}{2} \gamma-3\right)\left(1-\bar{x}^{2}\right) .
$$

Once we get the fixed points, we need to study the stability of the fixed point to ensure that the fixed points are actually giving us an attractor. If the fixed points are stable then we can have the attractor and finally we will be able to alleviate the 'cosmic coincidence' problem. To analyse the stability of these fixed points we perturb the system about the fixed point, $x \rightarrow \bar{x}+\delta x$ and $y \rightarrow \bar{y}+\delta y$. Then we study the evolution of the perturbations. If we have a growing solution of the perturbations our fixed points are not stable, however if we find a decaying solution we can say that our fixed points are stable. Substituting these values of $x$ and $y$ in equation (4.9) and (4.11) we get the perturbed equations of $x$ and $y$ as follows:

$$
\begin{gathered}
\delta x^{\prime}=\left[(\bar{\epsilon}-3)+(6-3 \gamma) \bar{x}^{2}+\frac{Q_{1}}{\bar{x}^{2}}-\frac{1}{\bar{x}} \frac{\partial Q_{1}}{\partial x}\right] \delta x- \\
\left(\frac{1}{\bar{x}} \frac{\partial Q_{1}}{\partial y}\right) \delta y, \\
\delta y^{\prime}=\left(\bar{\epsilon}+\frac{\lambda}{2 H}\right) \delta y .
\end{gathered}
$$

Here we have used $\delta \epsilon=[(6-3 \gamma) \bar{x}] \delta x$ and $\bar{y}=0$. Equations (5.7) and (5.8) can be written as

$$
\left(\begin{array}{l}
\delta x^{\prime} \\
\delta y^{\prime}
\end{array}\right)=(M)\left(\begin{array}{l}
\delta x \\
\delta y
\end{array}\right)
$$

where

$$
M=\left(\begin{array}{cc}
(\bar{\epsilon}-3)+(6-3 \gamma) \bar{x}^{2}+\frac{Q_{1}}{\bar{x}^{2}}-\frac{1}{\bar{x}} \frac{\partial Q_{1}}{\partial x} & \frac{1}{\bar{x}} \frac{\partial Q_{1}}{\partial y} \\
0 & \left(\bar{\epsilon}+\frac{\lambda}{2 H}\right)
\end{array}\right) .
$$

Two eigenvalues of the matrix $M$ are

$$
\mu_{1}=\left(\bar{\epsilon}+\frac{\lambda}{2 H}\right)
$$




$$
\mu_{2}=(\bar{\epsilon}-3)+(6-3 \gamma) \bar{x}^{2}+\frac{Q_{1}}{\bar{x}^{2}}-\frac{1}{\bar{x}} \frac{\partial Q_{1}}{\partial x} .
$$

Stability around the fixed points depend upon the nature of the eigen values $\mu_{1}$ and $\mu_{2}$. When $\mu_{1}<0, \mu_{2}<0$ the fixed points are stable and we can get an attractor solution. If $\mu_{1}>0, \mu_{2}>0$, the fixed points are unstable and we can not have any attractor. If one of them is positive and other one is negative, we get a saddle point which says that at one direction the fixed points are stable and at the other direction the fixed points are unstable.

In Ref.[21] it was noted that equation-of-state parameter $w_{\phi}=\frac{p_{\phi}}{\rho_{\phi}} \geq-1$ when $\dot{\phi}^{2} \geq$ $\frac{1}{4}\left(H \phi^{2}\right)$. The dark energy will enter the phantom region $\left(w_{\phi}<-1\right)$ if $\dot{\phi}^{2} \leq \frac{1}{4}\left(H \phi^{2}\right)$. Therefore in the region $w_{\phi} \geq-1$ we always get $X>0$. Now from Freidmann equation (4.4) we get

$$
H^{2}=\frac{8 \pi G}{3}\left(X+\tilde{V}+\rho_{m}\right),
$$

which implies that $H^{2}>\frac{8 \pi G}{3} \tilde{V}$. Finally taking logarithmic time derivative on both the sides of this inequality we get

$$
\epsilon+\frac{\lambda}{2 H}<0
$$

This means that $\mu_{1}$ is always negative as far as $w_{\phi} \geq-1$ is concerned. Therefore it is possible to have a stable fixed point if $\mu_{2}$ becomes negative for some interaction $Q$. In the next section, we analyse this for three types of interactions.

\section{$5.2 \quad$ Case II}

Substituting the value of $\bar{\epsilon}$ and constraint (4.5) in Eqs. (4.9) and (4.10), we get for $(\gamma=1)$ :

$$
\begin{aligned}
x^{\prime} & =(\bar{\epsilon}-3) x-\frac{\lambda}{H} \frac{y^{2}}{x}-\frac{Q_{1}}{x} \\
v^{\prime} & =\left(\bar{\epsilon}-\frac{3}{2}\right) v+\frac{Q_{1}}{v}
\end{aligned}
$$

Substituting for $\lambda$, we get,

$$
\begin{aligned}
\bar{\epsilon} & =-\frac{\lambda}{2 H}=3 x^{2}+\frac{3}{2} v^{2} \\
\delta \epsilon & =6 x \delta x+3 v \delta v
\end{aligned}
$$

The perturbed equations about the fixed point are:

$$
\begin{aligned}
& \delta x^{\prime}=\left(3-9 x^{2}-\frac{15}{2} v^{2}-3 \frac{v^{2}}{x^{2}}+3 \frac{v^{4}}{x^{2}}\right) \delta x+\left(6 \frac{v}{x}-12 \frac{v^{3}}{x}-15 x v\right) \delta v-\delta\left(\frac{Q_{1}}{x}\right) \\
& \delta v^{\prime}=6 x v \delta x+\left(3 x^{2}+\frac{9}{2} v^{2}-\frac{3}{2}\right) \delta v+\delta\left(\frac{Q_{1}}{v}\right)
\end{aligned}
$$

These attractor points are unique to ELKO cosmology regarding which we would like to stress the following points:

1. The perturbed equations do not explicitly depend on the potential. Hence, these equations can be realised for any potential provided $\bar{\varepsilon}=-\lambda /(2 H)$ is satified.

2. If $x \ll 1$ and $v \rightarrow 1$ (or vice-versa), Eq. (5.16) implies that $\epsilon>1$. It is easy to see that $\ddot{a}(t)>0$ implying that this corresponds to fast-roll inflation [28]. 


\section{Special cases of the interaction term}

In the previous section, we have obtained the condition for the existence of fixed point for general interaction. However, the analysis for a general interaction term is complicated. Here, for two cases, we take simple form of the interaction term and show explicitly the nature of the fixed points.

\subsection{Case I: Slow-roll}

\subsection{1 $Q_{1}=\beta v^{2} x$}

In this case the fixed point $\bar{y}$ is zero. The fixed point $\bar{x}$ and $\bar{v}$ can be found using the equation (5.1). Substituting $\bar{y}=0$ in equation (5.1) and using $\bar{v}^{2}=1-\bar{x}^{2}$ from equation (4.5) we can write

$$
(\bar{\epsilon}-3) \bar{x}-\frac{Q_{1}}{\bar{x}}=0 .
$$

Which gives us two solutions for $\bar{x}$

$$
\bar{x}= \pm 1, \quad \bar{x}=-\frac{\beta}{\left(3-\frac{3}{2} \gamma\right)} .
$$

Now $\bar{x}= \pm 1$ can not be a scaling solution because that will make our universe completely dark-energy dominated. Therefore the only possible solution is $\bar{x}=-\frac{\beta}{\left(3-\frac{3}{2} \gamma\right)}$ which is negative as $\beta$ and $\left(3-\frac{3}{2} \gamma\right)$ are both positive.

Therefore in this case the eigenvalue $\mu_{2}$ of the matrix $M$ can be written as

$$
\mu_{2}=\left[-\left(3-\frac{3}{2} \gamma\right)\left(1-3 \bar{x}^{2}\right)+2 \beta \bar{x}\right] .
$$

Now substituting the solution of $\bar{x}$ from (6.2) in the above expression of $\mu_{2}$ one can get the following expression of $\mu_{2}$ :

$$
\mu_{2}=-\left(3-\frac{3}{2} \gamma\right)+\frac{\beta^{2}}{\left(3-\frac{3}{2} \gamma\right)},
$$

From the above expression of $\mu_{2}$ it can be understood that when the first term dominates over the last term one can get $\mu_{2}<0$. Therefore the condition for having a stable fixed point for this kind of interaction is:

$$
\beta<\left(3-\frac{3}{2} \gamma\right)
$$

Therefore in this kind of interaction the coupling constant can not be very large and $\bar{\epsilon}$ is always less than 1 .

\subsection{2 $Q_{1}=\beta v^{2} x^{2}$}

In this case equation (5.6) and (6.1) tell us that

$$
\left(\frac{3}{2} \gamma-3\right)\left(1-\bar{x}^{2}\right) \bar{x}-\beta\left(1-\bar{x}^{2}\right) \bar{x}=0 .
$$

So, the only solution of $\bar{x}=(0, \pm 1)$. One can not take these solutions as fixed points as the universe will become purely matter dominated and dark-energy dominated respectively in those cases. Therefore we do not have any physical fixed points in this kind of interaction. 


\subsection{3 $Q_{1}=\beta v x^{2}$}

Following the similar method as described above using (6.1) for this kind of interaction one can find that at fixed point the only solution for $x$ is:

$$
\bar{x}= \pm \sqrt{1-\frac{\beta^{2}}{\left(3-\frac{3}{2} \gamma\right)^{2}}},
$$

Here we have considered $\bar{x} \neq(0, \pm 1)$. Substituting the above expression of $Q_{1}$ in the expression of $\mu_{2}$ one can get

$$
\mu_{2}=(6-3 \gamma) \bar{x}^{2}+\beta \frac{\bar{x}^{2}}{\bar{v}}
$$

Using the definition of $\bar{v}^{2}=1-\bar{x}^{2}$ and the expression of $\bar{x}$ from (6.7) one can write the expression of $\mu_{2}$ in terms of the coupling $\beta$ as:

$$
\mu_{2}=\left(3-\frac{3}{2} \gamma\right)-\frac{\beta^{2}}{\left(3-\frac{3}{2} \gamma\right)}
$$

Therefore in this case the $\mu_{2}$ will be negative only when $\beta>\left(3-\frac{3}{2} \gamma\right)$. However from (6.7) one can see that this condition will make $\bar{x}$ imaginary. Therefore we can not find a stable fixed point in this case.

\subsection{Case II: Fast roll}

\subsection{1 $Q_{1}=\beta v^{2} x$}

For this interaction, the perturbed equations of $x$ and $v$ are:

$$
\begin{aligned}
& \delta x^{\prime}=\left[3-9 x^{2}-\frac{15}{2} v^{2}-3 \frac{v^{2}}{x^{2}}+3 \frac{v^{4}}{x^{2}}\right] \delta x+\left[6 \frac{v}{x}-12 \frac{v^{3}}{x}-15 x v-2 \beta v\right] \delta v . \\
& \delta v^{\prime}=[6 x v+\beta v] \delta x+\left[3 x^{2}+\frac{9}{2} v^{2}-\frac{3}{2}+\beta x\right] \delta v
\end{aligned}
$$

The two eigen-values corresponding to the above set of equations are negative. Fig. (1a) shows that for different initial conditions $v \rightarrow 1$ and $x \ll 1$ is an attractor point.

\subsection{2 $Q_{1}=\beta v^{2} x^{2}$}

For this interaction, the perturbed equations of $x$ and $v$ are:

$$
\begin{aligned}
& \delta x^{\prime}=\left[3-9 x^{2}-\frac{15}{2} v^{2}-3 \frac{v^{2}}{x^{2}}+3 \frac{v^{4}}{x^{2}}-\beta v^{2}\right] \delta x+\left[6 \frac{v}{x}-12 \frac{v^{3}}{x}-15 x v-2 \beta v x\right] \delta v \\
& \delta v^{\prime}=[6 x v+2 \beta v x] \delta x+\left[3 x^{2}+\frac{9}{2} v^{2}-\frac{3}{2}+\beta x^{2}\right] \delta v
\end{aligned}
$$

Here again, both the eigenvalues corresponding to the above set of equations are negative. The eigenvalues are negative for all ranges of $\beta$ for which $x$ and $v$ are real. Fig. (1b) shows that for different initial conditions $v \rightarrow 1$ and $x \ll 1$ is an attractor point. 
6.2.3 $Q_{1}=\beta v x^{2}$

For this interaction, the perturbed equations of $x$ and $v$ are:

$$
\begin{aligned}
& \delta x^{\prime}=\left[3-9 x^{2}-\frac{15}{2} v^{2}-3 \frac{v^{2}}{x^{2}}+3 \frac{v^{4}}{x^{2}}-\beta v\right] \delta x+\left[6 \frac{v}{x}-12 \frac{v^{3}}{x}-15 x v-\beta x\right] \delta v . \\
& \delta v^{\prime}=[6 x v+2 \beta x] \delta x+\left[3 x^{2}+\frac{9}{2} v^{2}-\frac{3}{2}\right] \delta v
\end{aligned}
$$

Here again, both the eigenvalues corresponding to the above set of equations are negative for all valus of $\beta$ where $x$ and $v$ are real. Fig. (1c) shows that for different initial conditions $v \rightarrow 1$ and $x \ll 1$ is an attractor point.

\section{Conclusion}

As it is known, it is not possible to know precisely the initial condition of the field that drives inflation. Hence, any model of inflation need to show that attractor points exists in the space of the matter field variables. In all previous work, it was not possible to show explicitly that the Elko condensate does indeed lead to late-time attractor behavior.

In this work we have shown that rewritting the background field equations interms of new variables $X$ and $\tilde{V}$, one could show the existence of late-time attractor. Interestingly, we notice that the two set of attractor points exist for ELKO condensates. In case I, which is similar to canonical scalar field inflation [11], the attractors are realised only when $\bar{\epsilon}<1$. In case II, which is unique for ELKO cosmology, the attractors are realised only when $\bar{\epsilon}>1$ and they exist independent of the form of the potential. It will be interesting to repeat Starobinsky's analysis [28] for this case and see whether the spectrum of perturbations are nearly scale-invariant.

In the phantom region $\left(w_{\phi} \leq-1\right)$ obtaining stable fixed point may not be possible as

in that case $X<0$ may not allow $H^{2}>\tilde{V}$, in other words in phantom region $\mu_{1}<0$ may not be possible always. In this work the variables we have worked with are not a simple transformation of the variables chosen by Ref.[21]. The stability may be achieved by redefining the potential and the kinetic part. This is currently work under progress.

\section{Acknowledments}

SS acknowledges the support of DST, Government of India through Ramanujan fellowship and Max Planck-India Partner Group on Gravity and Cosmology. KVPV is supported by DST, Goverment of India through KVPY fellowship.

\section{References}

[1] A. H. Guth, The Inflationary Universe: A Possible Solution to the Horizon and Flatness Problems, Phys. Rev. D23 (1981) 347-356.

[2] A. D. Linde, Particle physics and inflationary cosmology, hep-th/0503203.

[3] E. W. Kolb, J. E. Lidsey, M. Abney, E. J. Copeland, and A. R. Liddle, The inflaton potential from present day observations, Nucl. Phys. Proc. Suppl. 43 (1995) 118-125.

[4] A. Golovnev, V. Mukhanov, and V. Vanchurin, Vector inflation, JCAP 0806 (2008) 009, [arXiv: 0802.2068]. 
[5] J. D. Barrow and S. Hervik, Simple Types of Anisotropic Inflation, Phys.Rev. D81 (2010) 023513, [arXiv:0911.3805].

[6] A. Golovnev, On cosmic inflation in vector field theories, Class.Quant.Grav. 28 (2011) 245018, [arXiv: 1109.4838].

[7] D. Gredat and S. Shankaranarayanan, Consistency relation between the scalar and tensor spectra in spinflation, JCAP 1001 (2010) 008, [arXiv:0807.3336].

[8] J. E. Lidsey, A. R. Liddle, E. W. Kolb, E. J. Copeland, T. Barreiro, and M. Abney, Reconstructing the inflaton potential: An overview, Rev. Mod. Phys. 69 (1997) 373-410, [astro-ph/9508078].

[9] B. A. Bassett, S. Tsujikawa, and D. Wands, Inflation dynamics and reheating, Rev.Mod.Phys. 78 (2006) 537-589, [astro-ph/0507632].

[10] K. A. Malik and D. Wands, Cosmological perturbations, Phys. Rept. 475 (2009) 1-51, [arXiv:0809.4944].

[11] E. J. Copeland, A. R. Liddle, and D. Wands, Exponential potentials and cosmological scaling solutions, Phys. Rev. D57 (1998) 4686-4690, [gr-qc/9711068].

[12] E. J. Copeland, D. J. Mulryne, N. J. Nunes, and M. Shaeri, Superinflation in loop quantum cosmology, Phys. Rev. D77 (Jan, 2008) 023510.

[13] D. V. Ahluwalia and D. Grumiller, Spin half fermions with mass dimension one: Theory, phenomenology, and dark matter, JCAP 0507 (2005) 012, [hep-th/0412080].

[14] D. V. Ahluwalia and D. Grumiller, Dark matter: A Spin one half fermion field with mass dimension one?, Phys.Rev. D72 (2005) 067701, [hep-th/0410192].

[15] C. G. Boehmer, The Einstein-Cartan-Elko system, Annalen Phys. 16 (2007) 38-44, [gr-qc/0607088].

[16] C. Boehmer, The Einstein-Elko system: Can dark matter drive inflation?, Annalen Phys. 16 (2007) 325-341, [gr-qc/0701087].

[17] C. G. Boehmer, J. Burnett, D. F. Mota, and D. J. Shaw, Dark spinor models in gravitation and cosmology, JHEP 1007 (2010) 053, [arXiv: 1003.3858].

[18] S. Shankaranarayanan, What-if inflaton is a spinor condensate?, Int.J.Mod.Phys. D18 (2009) 2173-2179, [arXiv:0905.2573].

[19] S. Shankaranarayanan, Dark spinor driven inflation, arXiv:1002.1128.

[20] A. Basak and J. R. Bhatt, Lorentz invariant dark-spinor and inflation, JCAP 1106 (2011) 011, [arXiv: 1104.4574].

[21] H. Wei, Spinor Dark Energy and Cosmological Coincidence Problem, Phys.Lett. B695 (2011) 307-311, [arXiv: 1002.4230].

[22] H. M. Sadjadi, On coincidence problem and attractor solutions in ELKO dark energy model, Gen.Rel.Grav. 44 (2012) 2329-2336, [arXiv:1109.1961].

[23] R. da Rocha and J. Hoff da Silva, ELKO, flagpole and flag-dipole spinor fields, and the instanton Hopf fibration, Adv.Appl.Clifford Algebras 20 (2010) 847-870, [arXiv:0811.2717].

[24] J. Hoff da Silva and R. da Rocha, From Dirac Action to ELKO Action, Int.J.Mod.Phys. A24 (2009) 3227-3242, [arXiv:0903.2815].

[25] L. Fabbri, Causality for ELKOs, Mod.Phys.Lett. A25 (2010) 2483-2488, [arXiv:0911.5304].

[26] L. Fabbri, Causal propagation for ELKO fields, Mod.Phys.Lett. A25 (2010) 151-157, [arXiv: 0911.2622]. 
[27] L. Fabbri, The Most General Cosmological Dynamics for ELKO Matter Fields, Phys.Lett. B704 (2011) 255-259, [arXiv:1011.1637].

[28] A. A. Starobinsky, Inflaton field potential producing the exactly flat spectrum of adiabatic perturbations, JETP Lett. 82 (2005) 169-173, [astro-ph/0507193]. 
This figure "Interaction1.png" is available in "png" format from: http://arxiv.org/ps/1212.3445v1 
This figure "Interaction2.png" is available in "png" format from: http://arxiv.org/ps/1212.3445v1 
This figure "Interaction3.png" is available in "png" format from: http://arxiv.org/ps/1212.3445v1 